\title{
Growth of graph powers
}

\author{
A. Pokrovskiy * \\ Departement of Mathematics \\ London School of Economics and Political Sciences, \\ Houghton Street, WC2A 2AE \\ London, United Kingdom. \\ a.pokrovskiy@lse.ac.uk
}

Submitted: Nov 11, 2010; Accepted: Mar 23, 2011; Published: Apr 14, 2011

Mathematics Subject Classification: $05 \mathrm{C} 12$

\begin{abstract}
For a graph $G$, its $r$ th power is constructed by placing an edge between two vertices if they are within distance $r$ of each other. In this note we study the amount of edges added to a graph by taking its $r$ th power. In particular we obtain that, for $r \geq 3$, either the $r$ th power is complete or "many" new edges are added. In this direction, Hegarty showed that there is a constant $\epsilon>0$ such $e\left(G^{3}\right) \geq(1+\epsilon) e(G)$. We extend this result in two directions. We give an alternative proof of Hegarty's result with an improved constant of $\epsilon=\frac{1}{6}$. We also show that for general $r$, $e\left(G^{r}\right) \geq\left(\left\lceil\frac{r}{3}\right\rceil-1\right) e(G)$.
\end{abstract}

\section{Introduction}

This note addresses some questions raised by P. Hegarty in [4]. In that paper he studied results about graphs inspired by the Cauchy-Davenport Theorem.

All graphs in this paper are simple and loopless. For two vertices $u, v \in V(G)$, denote the length of the shortest path between them by $d(u, v)$. For $v \in V(G)$, define its ith neighborhood as $N_{i}(v)=\{u \in V(G): d(u, v)=i\}$. The $r$ th power of a graph $G$, denoted $G^{r}$, is constructed from $G$ by adding an edge between two vertices $x$ and $y$ when they are within distance $r$ in $G$. Define the diameter of $G, \operatorname{diam}(G)$, as the minimal $r$ such that $G^{r}$ is complete (alternatively, the maximal distance between two vertices). Denote the number of edges of $G$ by $e(G)$. For $v \in V(G)$ and a set of vertices $S$, define $e^{r}(v, S)=|\{u \in S: d(v, u) \leq r\}|$.

The Cayley graph of a subset $A \subseteq \mathbb{Z}_{p}$ is constructed on the vertex set $\mathbb{Z}_{p}$. For two distinct vertices $x, y \in \mathbb{Z}_{p}$, we define $x y$ to be an edge whenever $x-y \in A$ or $y-x \in A$.

\footnotetext{
${ }^{*}$ Research supported by the LSE postgraduate research studentship scheme.
} 
The following is a consequence of the Cauchy-Davenport Theorem (usually stated in the language of additive number theory $[1,2])$.

Theorem 1. Let $p$ be a prime, $A$ a subset of $\mathbb{Z}_{p}$, and $G$ the Cayley graph of $A$. Then for any integer $r<\operatorname{diam}(G)$ :

$$
e\left(G^{r}\right) \geq \operatorname{re}(G)
$$

If we take $A$ to be the arithmetic progression $\{a, 2 a, \ldots, k a\}$, then equality holds in this theorem for all $r<\operatorname{diam}(G)$. We might look for analogues of Theorem 1 for more general graphs $G$. In particular since these Cayley graphs are always regular and (when $p$ is prime) connected, we might focus on regular, connected G. In [4] Hegarty proved the following theorem:

Theorem 2. Suppose $G$ is a regular, connected graph with $\operatorname{diam}(G) \geq 3$. Then we have

$$
e\left(G^{3}\right) \geq(1+\epsilon) e(G)
$$

with $\epsilon \approx 0.087$

In other words, the cube of $G$ retains the original edges of $G$ and gains a positive proportion of new ones. In Section 3 we prove this theorem with an improved constant of $\epsilon=\frac{1}{6}$. Since we announced this note, DeVos and Thomassé [3] further improved the constant in Theorem 2 to $\epsilon=\frac{3}{4}$. They also show that the constant cannot be improved further by exhibiting a sequence of regular graphs $G_{n}$, such that $\frac{e\left(G_{n}^{r}\right)}{e\left(G_{n}\right)} \rightarrow \frac{7}{4}$ as $n \rightarrow \infty$.

Theorem 2 leads to the question of how the growth behaves for other powers of the $G$. Note that Theorem 2 cannot be used recursively to obtain such a result - since the cube of a regular graph is not necessarily regular. In [4] it was shown that no equivalent of Theorem 2 exists with $G^{3}$ replaced by $G^{2}$, and it was asked what happens for higher powers. In this note we address that question.

\section{Main Result}

We prove the following theorem:

Theorem 3. Suppose $G$ is a regular, connected graph, and $r \leq \operatorname{diam}(G)$. Then we have:

$$
e\left(G^{r}\right) \geq\left(\left\lceil\frac{r}{3}\right\rceil-1\right) e(G)
$$

Proof. Let the degree of each vertex be $d$. Fix some $v$ with $N_{\operatorname{diam}(G)}(v)$ nonempty.

Consider any vertex $u \in V(G)$. Then for any $j$ satisfying $d(u, v)-r<j \leq d(u, v)$, there is a $w_{j} \in N_{j}(v)$ such that $d\left(u, w_{j}\right)<r$. For such a $w_{j}$, all vertices $x \in N_{1}\left(w_{j}\right)$ have $d(u, x) \leq r$. All such $x$ are contained in $N_{j-1}(v) \cup N_{j}(v) \cup N_{j+1}(v)$, hence

$$
e^{r}\left(u, N_{j-1}(v) \cup N_{j}(v) \cup N_{j+1}(v)\right) \geq d .
$$


Note that each $j \in\left\{d(u, v)-3, d(u, v)-6, \ldots, d(u, v)-3\left(\left\lceil\frac{1}{3} \min \{d(u, v), r\}\right\rceil-1\right)\right\}$ satisfies $d(u, v)-r<j \leq d(u, v)$. Summing the bound (1) over all these $j$, noting that any edge is counted at most once, we obtain

$$
e^{r}\left(u, N_{0}(v) \cup \cdots \cup N_{d(u, v)-2}(v)\right) \geq\left\lceil\frac{1}{3} \min \{d(u, v), r\}\right\rceil d-d .
$$

Now we sum this over all $u \in G$. Note that since the edges counted above go from some $N_{i}(v)$ to $N_{j}(v)$ with $j<i$, each edge is counted at most once. Also we haven't yet counted any of the original edges of $G$, so we might as well add them. Hence

$$
\begin{aligned}
e\left(G^{r}\right) & \geq \sum_{u \in G} e^{r}\left(u, N_{0}(v) \cup \cdots \cup N_{d(u, v)-2}(v)\right)+e(G) \\
& \geq \sum_{u \in G}\left\lceil\frac{1}{3} \min \{d(u, v), r\}\right] d-|V(G)| d+e(G) \\
& =\sum_{u \in G}\left\lceil\frac{1}{3} \min \{d(u, v), r\}\right] d-e(G) .
\end{aligned}
$$

Obviously there was nothing particularly special about $v$. We can get a similar expresssion using $v^{\prime} \in N_{\operatorname{diam}(G)}(v)$, namely

$$
e\left(G^{r}\right) \geq \sum_{u \in G}\left\lceil\frac{1}{3} \min \left\{d\left(u, v^{\prime}\right), r\right\}\right\rceil d-e(G) .
$$

Averaging (2) and (3) we get

$$
e\left(G^{r}\right) \geq \frac{1}{2} \sum_{u \in G}\left(\left\lceil\frac{1}{3} \min \{d(u, v), r\}\right\rceil+\left\lceil\frac{1}{3} \min \left\{d\left(u, v^{\prime}\right), r\right\}\right\rceil\right) d-e(G) .
$$

Note that for any $u \in V(G)$ we have

$$
\left\lceil\frac{1}{3} \min \{d(u, v), r\}\right\rceil+\left\lceil\frac{1}{3} \min \left\{d\left(u, v^{\prime}\right), r\right\}\right\rceil \geq\left\lceil\frac{r}{3}\right\rceil .
$$

This is because $d(u, v)+d\left(u, v^{\prime}\right) \geq d\left(v, v^{\prime}\right)=\operatorname{diam}(G) \geq r$. Putting the bound (5) into the sum (4) we obtain

$$
e\left(G^{r}\right) \geq \frac{|V(G)| d}{2}\left\lceil\frac{r}{3}\right\rceil-e(G)=\left\lceil\frac{r}{3}\right\rceil e(G)-e(G) .
$$

Thus the theorem is proven.

\section{Cubes}

Note that for $r \leq 6$ the bounds in Theorem 3 are trivial. In particular it says nothing about the increase in the number of edges of the cube of a regular, connected graph. Such an increase was already demonstrated by Hegarty in Theorem 2. Here we give an alternative proof of that theorem, yielding a slightly better constant. 
Theorem 4. Suppose $G$ is a regular, connected graph with $\operatorname{diam}(G) \geq 3$. Then we have

$$
e\left(G^{3}\right) \geq\left(1+\frac{1}{6}\right) e(G) .
$$

Proof. Let the degree of each vertex be $d$. Note that as $G$ is regular, and not complete, every $v \in V(G)$ will have a non-neighbour in $G$. Together with connectedness this implies that each $v \in V(G)$ has at least one new neighbour in $G^{2}$. This implies the theorem for $d \leq 6$. For the remainder of the proof, we assume that $d>6$. The proof rests on the following colouring of the edges of $G$ : For an edge $u v$ in $G$, colour

$$
\begin{aligned}
u v \text { red if }\left|N_{1}(u) \cap N_{1}(v)\right| & >\frac{2}{3} d, \\
u v \text { blue if }\left|N_{1}(u) \cap N_{1}(v)\right| & \leq \frac{2}{3} d .
\end{aligned}
$$

Notice that if $u v$ is a blue edge, then there are at least $\frac{4}{3} d-1$ neighbours of $u$ in $G^{2}$. This is because $u$ will be connected to everything in $N_{1}(u) \cup N_{1}(v)$ except itself, and $\left|N_{1}(u) \cup N_{1}(v)\right| \geq \frac{4}{3} d$ for $u v$ blue. If, in addition, we have some $x$ connected to $u$ by an edge (of any colour), then $x$ will be at distance at most 3 from everything in $N_{1}(u) \cup N_{1}(v) \backslash\{x\}$. Hence $x$ will have at least $\frac{4}{3} d-1$ neighbours in $G^{3}$.

Partition the vertices of $G$ as follows:

$B=\{v \in V(G): v$ has a blue edge coming out of it $\}$,

$R=\{v \in V(G): v \notin B$ and there is a $u \in B$ such that $u v$ is an edge $\}$,

$S=V(G) \backslash(B \cup R)$.

By the above argument, if $v$ is in $B \cup R$, then $e^{3}(v, V(G)) \geq \frac{4}{3} d-1$. Recall that each $u \in S$ will have at least one new neighbour in $G^{2}$, giving $e^{3}(u, V(G)) \geq d+1$. Summing these two bounds over all vertices in $G$, noting that any edge is counted twice, gives

$$
\begin{aligned}
2 e\left(G^{3}\right) & \geq\left(\frac{4}{3} d-1\right)|B \cup R|+(d+1)|S| \\
& =\left(\frac{4}{3} d-1\right)|B \cup R|+(d+1)(|V(G)|-|B \cup R|) \\
& =\frac{7}{6} d|V(G)|+\frac{1}{3}\left(|B \cup R|-\frac{1}{2}|V(G)|\right)(d-6) \\
& =\frac{7}{3} e(G)+\frac{1}{3}\left(|B \cup R|-\frac{1}{2}|V(G)|\right)(d-6) .
\end{aligned}
$$

Recall that we are considering the case when $d>6$. Thus to prove that $e\left(G^{3}\right) \geq \frac{7}{6} e(G)$, it suffices to show that $|B \cup R| \geq \frac{1}{2}|V(G)|$. To this end we shall demonstrate that $|S| \leq|R|$. First however we need a proposition helping us to find blue edges in $G$.

Proposition 5. For any $v \in V(G)$ there is some $b \in B$ such that $d(v, b) \leq 2$. 
Proof. Suppose $d(v, u)=3$. Then there are vertices $x$ and $y$ such that $\{v, x, y, u\}$ forms a path between $u$ and $v$. We will show that one of the edges $v x, x y$ or $y u$ is blue. This will prove the proposition assuming that there are any blue edges to begin with. However, it also shows the existence of blue edges because $\operatorname{diam}(G) \geq 3$.

So, suppose that the edges $v x$ and $u y$ are red. Then we have $\left|N_{1}(v) \cap N_{1}(x)\right|>\frac{2}{3} d$, and $\left|N_{1}(u) \cap N_{1}(y)\right|>\frac{2}{3} d$. Using this and $N_{1}(u) \cap N_{1}(v)=\emptyset$ gives

$$
\begin{aligned}
\left|N_{1}(x) \cup N_{1}(y)\right| & \geq\left|\left(N_{1}(x) \cup N_{1}(y)\right) \cap N_{1}(v)\right|+\left|\left(N_{1}(x) \cup N_{1}(y)\right) \cap N_{1}(u)\right| \\
& \geq\left|N_{1}(x) \cap N_{1}(v)\right|+\left|N_{1}(y) \cap N_{1}(u)\right| \\
& >\frac{4}{3} d .
\end{aligned}
$$

Therefore $\left|N_{1}(x) \cap N_{1}(y)\right|=2 d-\left|N_{1}(x) \cup N_{1}(y)\right| \leq \frac{2}{3} d$. Hence $x y$ is blue, proving the proposition.

Now we will show that $|S| \leq|R|$. Suppose $r \in R$. By the definition of $R$, there is a $b \in B$ such that $r b$ is an edge. This edge is neccesarily red as $r \notin B$. Using $N_{1}(b) \subseteq B \cup R$, we have $\left|N_{1}(r) \cap(B \cup R)\right| \geq\left|N_{1}(r) \cap N_{1}(b)\right|>\frac{2}{3} d$. Hence

$$
\left|N_{1}(r) \cap S\right| \leq \frac{1}{3} d
$$

Suppose $s \in S$. Proposition 5 implies that there is some $r \in R$ such that $s r$ is an edge. Since $s r$ is red, we have $\left|N_{1}(s) \cap N_{1}(r)\right|>\frac{2}{3} d$. Using this, the fact that $N_{1}(s) \subseteq R \cup S$, and (6), gives

$$
\begin{aligned}
\left|N_{1}(s) \cap R\right| & \geq\left|N_{1}(s) \cap N_{1}(r) \cap R\right| \\
& =\left|N_{1}(s) \cap N_{1}(r)\right|-\left|N_{1}(s) \cap N_{1}(r) \cap S\right| \\
& \geq\left|N_{1}(s) \cap N_{1}(r)\right|-\left|N_{1}(r) \cap S\right| \\
& >\frac{1}{3} d .
\end{aligned}
$$

Double-counting the edges between $S$ and $R$ using the bounds (6) and (7) gives a contradiction unless $|S| \leq|R|$. Therefore $|B \cup R| \geq \frac{1}{2}|V(G)|$ as required.

\section{Discussion}

Theorem 3 answers the question of giving a lower bound on the number of edges that are gained by taking higher powers of a graph. We obtain growth that is linear with $r$ - just as in Theorem 1.

- The constant $\left\lceil\frac{1}{3} r\right\rceil$ in Theorem 3 cannot be improved to something of the form $\lambda r$ with $\lambda>\frac{1}{3}$. To see this, consider the following sequence of graphs $H_{r}(d)$ as $d$ tends to infinity: 


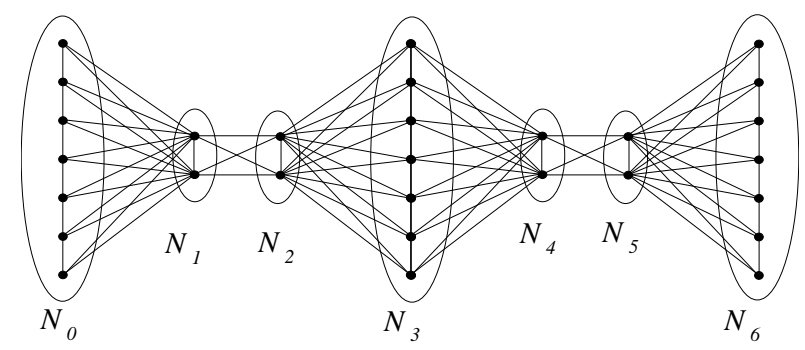

Figure 1: The graph $H_{6}(8)$.

Take disjoint sets of vertices $N_{0}, \ldots, N_{r}$, with $\left|N_{i}\right|=d-1$ if $i \equiv 0(\bmod 3)$ and $\left|N_{i}\right|=2$ otherwise. Add all the edges within each set and also between neighboring ones. So if $u \in N_{i}, v \in N_{j}$, then $u v$ is an edge whenever $|i-j| \leq 1$ (see Figure 1).

The number of edges in $H_{r}(d)$ is at least the number of edges in the larger classes which is $\left\lceil\frac{1}{3}(r+1)\right\rceil\left(\begin{array}{c}d-1 \\ 2\end{array}\right)$.

The $r$ th power $H_{r}(d)^{r}$ has less than $\left(\begin{array}{c}|V(G)| \\ 2\end{array}\right)$ edges which is less than $\left(\begin{array}{c}\left\lceil\frac{1}{3}(r+1)\right\rceil(d+3) \\ 2\end{array}\right)$. Therefore,

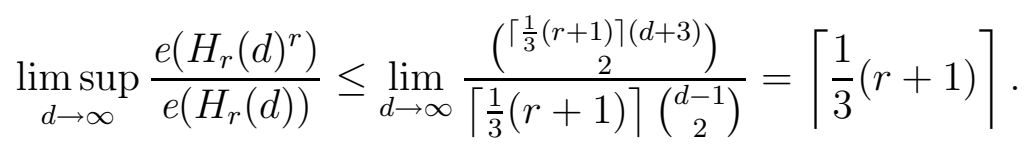

The graphs $H_{r}(d)$ are not regular, but if $r \not \equiv 2(\bmod 3)$, it is possible to remove a small (less than $|V(G)|$ ) number of edges from the graphs and make them $d$ regular without losing connectedness (any cycle passing through all the vertices in $N_{1} \cup \ldots \cup N_{r-1}$ would work). Call these new graphs $\hat{H}_{r}(d)$. By the same argument as before we have

$$
\limsup _{d \rightarrow \infty} \frac{e\left(\hat{H}_{r}(d)^{r}\right)}{e\left(\hat{H}_{r}(d)\right)} \leq\left\lceil\frac{1}{3}(r+1)\right\rceil .
$$

If $r \equiv 2(\bmod 3)$, a similar trick can be performed, but we'd need to start with $\left|N_{i}\right|=d-1$ if $i \equiv 1(\bmod 3)$ and $\left|N_{i}\right|=2$ otherwise.

So the factor of $\frac{1}{3}$ cannot be improved for regular graphs. All these examples are inspired by one given in [4] to show that for any $\epsilon$ there are regular graphs $G$ with $e\left(G^{2}\right)<(1+\epsilon) e(G)$.

- All the questions from this paper and [4] could be asked for directed graphs. In particular one can define directed Cayley graphs for a set $A \subseteq \mathbb{Z}_{p}$ by letting $x y$ be a directed edge whenever $x-y \in A$. Then the Cauchy-Davenport Theorem implies an identical version of Theorem 1 for directed Cayley graphs. In this setting it is easy to show that there is growth even for the square of an out-regular oriented graph $D$ (a directed graph where for a pair of vertices $u$ and $v, u v$ and $v u$ are not 
both edges). In particular, we have

$$
e\left(D^{2}\right) \geq \frac{3}{2} e(D)
$$

This occurs because every vertex $v$ has $\left|N_{2}^{\text {out }}(v)\right| \geq \frac{1}{2}\left|N_{1}^{\text {out }}(v)\right|$ in an out-regular oriented graph. It's easy to see that this is best possible for such graphs. One can construct out-regular oriented graphs with an arbitrarily large proportion of vertices $v$ satisfying $\left|N_{2}^{\text {out }}(v)\right|=\frac{1}{2}\left|N_{1}^{\text {out }}(v)\right|$.

However if we insist on both in and out-degrees to be constant, (8) no longer seems tight. Such graphs are always Eulerian. In [5] there is a conjecture attributed to Jackson and Seymour that if an oriented graph $D$ is Eulerian, then $e\left(D^{2}\right) \geq 2 e(D)$ holds. If this conjecture were proved, it would be an actual generalization of the directed version of Theorem 1, as opposed to the mere analogues proved above.

\section{Acknowledgement}

The author would like to thank his supervisors Jan van den Heuvel and Jozef Skokan for helpful advice and discussions.

\section{References}

[1] A. L. Cauchy. Recherches sur les nombres. J. Ecole Polytech, 9:99-116, 1813.

[2] H. Davenport. On the addition of residue classes. J. London Math. Soc., 10:30-32, 1935.

[3] M. DeVos and S. Thomassé. Edge growth in graph cubes. arXiv:1009.0343v1 [math.CO], 2010.

[4] P. Hegarty. A Cauchy-Davenport type result for arbitrary regular graphs. Integers, 11, Paper A19, 2011.

[5] B. D. Sullivan. A summary of results and problems related to the Caccetta-Häggkvist conjecture. AIM Preprint, 2006. 